\title{
Partly segregated cortico-subcortical pathways support phonologic and semantic verbal fluency: a lesion study
}

\author{
Leila Chouiter ${ }^{1,3}$, Josefina Holmberg ${ }^{1}$, Aurelie L. Manuel ${ }^{2}$, Françoise Colombo ${ }^{3}$, Stephanie \\ Clarke $^{2}$, Jean-Marie Annoni ${ }^{1}$, and Lucas Spierer ${ }^{1, *}$
}

\section{Affiliations:}

1. Neurology Unit, Department of Medicine, Faculty of Sciences, University of Fribourg, Fribourg, Switzerland

2. Neuropsychology and Neurorehabilitation Service, Department of Clinical Neurosciences, Vaudois University Hospital Center, Lausanne, Switzerland

3. Neuropsychology Unit, Hôpital fribourgeois, Fribourg, Switzerland

\author{
*Corresponding author: \\ PD Dr Lucas Spierer \\ Laboratory for Cognitive and Neurological Sciences \\ Neurology Unit, Medicine Department, University of Fribourg \\ Ch. du Musée 5, 1700 Fribourg, Switzerland \\ Ph : +41263008548; email : Lucas.spierer@unifr.ch
}

\section{Author Contributions:}

Study concept and design: LC; JMA; LS

Acquisition of data: LC; SC; FC; AM

Analysis and interpretation: JH; LC; LS; JMA

\section{Acknowledgements}

This work was supported by grants from the Swiss National Science Foundation (Grant \#32003B_156854 to LS; \#325100-118362 to JMA; and \#320030B-141177 to SCL). We thank Narges Radman for her help in collecting and analyzing the data and Philippe Maeder for providing us with an access to the radiologic data. 


\section{ABSTRACT}

Verbal fluency refers to the ability to generate as many words as possible in a limited time interval, without repetition and according to either a phonologic (each word begins with a given letter) or a semantic rule (each word belongs to a given semantic category). While current literature suggests the involvement of left fronto-temporal structures in fluency tasks, whether the same or distinct brain areas are necessary for each type of fluency remains unclear.

We tested the hypothesis for an involvement of partly segregated cortico-subcortical structures between phonologic and semantic fluency by examining with a voxel-based lesion symptom mapping approach the effects of brain lesions on fluency scores corrected for age and education level in a group of 191 unselected brain-damaged patients with a first left or right hemispheric lesion.

There was a positive correlation between the scores to the two types of fluency, suggesting that common mechanisms underlie the word generation independently of the production rule. The lesion symptom mapping revealed that lesions to left basal ganglia impaired both types of fluency and that left superior temporal, supramarginal and rolandic operculum lesions selectively impaired phonologic fluency and left middle temporal lesions impaired semantic fluency.

Our results corroborate current neurocognitive models of word retrieval and production, and refine the role of cortical-subcortical interaction in lexical search by highlighting the common executive role of basal ganglia in both types of verbal fluency and the preferential involvement of the ventral and dorsal language pathway in semantic and phonologic fluency, respectively.

\section{Keywords:}

Language, Executive functions, Verbal fluency, Brain lesion, Basal ganglia 


\section{INTRODUCTION}

Verbal fluency -or word generation- tasks are classically used for the neuropsychological assessment of language and executive functions (Moscovitch, 1994). Fluency tasks consist in generating as many words as possible over a given time interval, without repetition and according to either a phonologic (each word begins with a given letter) or a semantic rule (each word belongs to a given semantic category, as e.g. animal or fruit (Bechtoldt, 1962, Hodges et al., 1992, Tombaugh et al., 1999).

Verbal fluency not only requires accessing and retrieving specific words within lexical memory, but also monitoring responses to avoid repetitions and suppressing task-irrelevant words to stick to the task rules. These tasks thus involve language processing and the three components of the Miyake's model of executive functions ('Shifting', 'Updating' and 'Inhibiting'; (Miyake et al., 2000). Importantly, while both semantic and phonologic fluency involve a mnesic-associative and an executive component, their relative contribution differs between the two types of fluency. Retrieving words belonging to a given semantic category can indeed be achieved based on the default semantic organization of conceptual knowledge (Shapira-Lichter et al., 2013): participants might rely on association chains between items in a given category based on the fact that the brain activity associated with finding a first item could spread to other items of the same category (Gruenewald and Lockhead, 1980). In contrast, phonologic fluency requires inhibiting the default semantic associations to search words according to the unusual 'first letter' association between them. Phonologic fluency has thus been advanced to load more strongly on the executive component than semantic fluency (Perret, 1974; Thompson-Schill et al., 1997; ThompsonSchill et al., 1998; Katzev et al., 2013), although control processes are also likely necessary in semantic fluency to shift between subcategories of items and resist the interferences from competing alternatives when a given semantic network is activated (Mummery et al., 1996; Reverberi et al., 2006).

Since current neurocognitive models of verbal fluency assume that different processes and strategies are involved in semantic and phonologic fluency, these two tasks should rely on partly segregated brain networks. These models are supported by functional neuroimaging evidence for a prominent role of left frontal executive regions in phonologic fluency (Mummery et al., 1996, Pujol et al., 1996, Phelps et al., 1997), and of temporal associative areas during semantic fluency (Martin et al., 1996, Gourovitch et al., 2000). However, neuropsychological literature reports a slightly different pattern of difference between the neural correlates of the two types of fluency. A meta-analysis of 32 
neuropsychological studies including tests of verbal fluency in patients with focal brain damage indeed reports that while temporal structures are more important for semantic fluency, frontal damages impact similarly on phonologic and semantic fluency (Henry and Crawford, 2004). Of note, dorsal/ventral dissociations for phonologic and semantic processing have also been found related to other types of language impairments; deficits in oral expression can for example occur at the lexical-semantic or lexicalphonological levels (Henseler et al., 2014, Parker Jones et al., 2014).

Frontal and temporal areas have also been advanced to be involved in both types of fluency by studies focusing on cluster-switch behaviors; this concept refers to a word-retrieval strategy generally at play during verbal fluency tasks, which consists in generating words belonging to a given subcategory and then shifting between subcategories. According to this framework, frontal areas are suggested to be involved in switching and temporal areas in sweeping within a semantic or phonological field (e.g. Troyer et al. 1998).

Because of their connections to the cortical structures supporting verbal fluency, basal ganglia have also been involved in word production tasks (Fu et al., 2002). In the ganglia-thalamo-cortical loops, the DLPFC is connected to the dorsolateral caudate nucleus and the internal globus pallidus. Fluency impairments following basal ganglia disruption have notably been demonstrated in clinical studies on HIV (Thames et al., 2012), as well as in Huntington and Parkinson patients (Lawrence et al., 1998, Benke et al., 2003), and might follow from a disruption of the maintenance, monitoring and selection of goalrelevant representations by prefrontal cortices (Wagner et al., 2001).

Critically, current lesion data are undermined by a high degree of inconsistency in the effects of lesions on verbal fluency performance. Discrepancies on the neural underpinnings of fluency in neuropsychological studies most likely follow from the fact that the lesion studies having tested the two types of fluency in the same patients included small sample sizes (e.g. 32 in Martin et al., 1990, 32 in Vilkki and Holst, 1994, 12 in Baldo and Shimamura, 1998). In addition to limiting the statistical power of the analyses, small sample sizes tend to reduce the portion of the brain in which the effects of lesion are tested, leaving unresolved the role of many brain areas (e.g. Baldo et al. (2006), which included only lefthemispheric patients). Moreover, in most of previous lesion studies on verbal fluency, patients were selected based on a priori hypotheses on the role of specific brain regions or on the association between verbal fluency and specific neuropsychological syndromes (e.g. studies with aphasic patients in Grossman, 1981, frontal or temporal patients in Troyer et al., 1998, cortical lesions in Henry and Crawford, 2004, or left hemispheric patients in Baldo et al., 2006). 
With the aim of identifying the brain structures whose integrity is necessary for phonologic and/or semantic fluency, we analyzed statistically the relationship between verbal fluency performance and focal lesion locations using Voxel-based Lesion Symptom Mapping (VLSM; Bates et al., 2003). In contrast to functional neuroimaging approach, the analysis of lesion allows to establish causal relationships between brain and behavior and not only correlational associations between activity in a given brain area and performance at a given task.

To prevent selection biases, we opted for the most data-driven approach as possible by focusing on an unselected group of hemispheric brain damaged patients without any exclusion criteria at the level of lesion site or clinical profile. Most notably, we included both left and right hemispheric patients; while the prominent involvement of left hemispheric structure in fluency is clinically obvious, there is indeed a lack of direct empirical evidence for this question. Moreover, we included a very large sample of 191 patients to optimize the statistical sensitivity and brain coverage of our analyses. Because age and education level have been shown to influence fluency performance (Marsolais et al., 2015; Katzev et al., 2013; Tombaugh et al., 1999), we used the continuous fluency scores corrected for these factors as behavioral inputs in the analyses. We further analyzed the correlation between the score at each of the fluency task.

\section{MATERIAL AND METHODS}

\section{Population}

Hundred and ninety-one in-patients from the Neuropsychology departments of the Hôpital Fribourgeois and the Centre Hospitalier Universitaire Vaudois were included retrospectively in the study. All the patients were hospitalized between 2007 and 2015 for a first unilateral hemispheric lesion. The patients were aged $62.2 \pm 14.9$ years (mean $\pm S D$ ) and the group included a total of 71 women (see Table 1 for detailed demographic information). The routine neuropsychological assessment including the fluency tasks was conducted $2.4 \pm 5.3$ weeks (mean $\pm S D$ ) after the stroke onset or the diagnosis or ablation of the tumor. Inclusion criteria were: (i) first unilateral hemispheric lesion without damage to the brainstem or cerebellum documented by CT-scan and/or MRI (we excluded patients with bilateral lesions to enable interpreting our results in terms of hemispheric specialization); (ii) no prior neurological or psychiatric illness; (iii) sufficient understanding of the instructions (as clinically assessed by the neuropsychologists); (iv) assessment of both semantic and phonologic fluency; (v) right-handedness. We did not use lesion 
characteristics or patterns of behavioral deficit as inclusion criteria. The study was approved by our local Ethics Committee.

Table 1 near here

\section{Neuropsychological assessment of Phonologic and Semantic Verbal Fluency}

Phonologic fluency was assessed by asking the patients to report in one minute all the words they could starting with the sound " $\mathrm{M}$ " (132 patients) or " $\mathrm{S}$ " (59 patients); the choice of the letter was made arbitrarily by the clinician; there was no difference in the performance between the two letters $(t(189)=-$ 1.719; $p=0.09$ ). Semantic fluency was assessed by asking the patients to report in one minute as many animals as they could. The patients were instructed that they could tell any French word except private names and that repetitions were not allowed. Two to three examples were given before starting the phonologic fluency task (with another letter than $\mathrm{M}$ or S). The total number of correct words was calculated. Because the age and the educational level have been shown to impact the performance of the production in verbal fluency tests (Cardebat et al., 1990, Ruff et al., 1997, Tombaugh et al., 1999), the scores were corrected according to norms collected on healthy controls matched in age and educational level (Thuillard and Assal, 1991). We used data from two groups of age: 20-69 and from 70years old. These populations were also split based on their education level, with level $1=1-9$ years of school (mandatory school in Switzerland); level 2= 9-12 years (e.g. high-school); level 3= 12- years (e.g. university). The following corrections were then applied: For age-group 1, the correction was: Level 1, + 2 points to raw score; Level 2, +1 point; Level 3, - 4 points. For age-group 2, with: Level 1, + 6 points; Level 2, +2 points; Level 3, we didn't change the score. We opted for this approach rather than for regressing age and education level as nuisance covariates during the VLSM analyses because an interaction between these two factors, and non-linear relationships between these factors and the fluency scores cannot be excluded.

\section{Voxel-based statistical analyses of anatomo-functional correlations}

Voxel-based lesion-symptom mapping is a method enabling the statistical assessment of anatomoclinical correlations by comparing the performance of patients at a given neuropsychological test between those with vs. without a lesion at across the brain with a high spatial resolution (Bates et al., 
2003). Brain lesions from each patient were drawn on the standard Montreal Neurological Institute's (MNI) brain template by a trained assistant naive to the patients' neuropsychological profile using the MRICro software (Rorden and Brett, 2000, for similar approaches see Spierer et al., 2009, or Manuel et al., 2013). The manual reconstruction of the lesions on a template has been demonstrated to be as accurate as automatic or semi-automatic reconstruction in two comparative studies (Fiez et al., 2000, Wilke et al., 2011). We would further note that the manual procedure was well adapted to the present retrospective study in which lesion reconstructions were based on clinical radiologic data that were not acquired for research purposes and thus showed variable acquisition parameters. To identify brain areas whose integrity impacted on verbal fluency performance, the lesions and fluency scores were submitted to statistical mapping analyses using the voxel-wise lesion-symptom mapping (VLSM) algorithms implemented in the NPM software by Rorden et al. (2007). The VLSM was based on the continuous fluency scores corrected for education level and age. T-tests on the continuous corrected verbal fluency scores were run separately on each voxel to compare performance in patients with vs. without lesion at this voxel. The statistical tests were conducted only on voxels damaged in at least 6 patients; this threshold was chosen to ensure a minimal statistical power while covering with the tests a portion of the brain as large as possible (Rorden et al., (2007); fig. 2A). The results of the t-tests were then color-coded and projected on the MNI template brain using MRICro (Rorden and Brett, 2000) for voxels surviving a significance threshold of $p<0.05$ with a False Discovery Rate (FDR) correction.

\section{RESULTS}

There was a positive correlation between the performance in the phonologic and semantic fluency task $(r(189)=0.704 ; p<0.001 ;$ Figure 1). The performance at the semantic fluency task was better than at the phonologic fluency $(t(190)=19.009 ; p<0.001)$.

As a control analysis, we examined the relationship between lesion volume and the fluency scores. We found no evidence for correlation between total lesion volume and the fluency scores (Phonologic fluency: $r(189)=-0.102 ; p=0.16$; Semantic Fluency: $r(189)=-0.021 ; p=0.77)$, suggesting that this factor did not confound our result.

The VLSM results revealed that both shared and specific brain areas were involved in phonologic and semantic fluency. 
Both phonologic and semantic fluency were associated with left subcortical areas including the putamen, caudate nucleus and pallidum, as well as to left cortical areas including the superior and middle temporal gyri, the angular gyri, the insula and parts of the supramarginal gyri. The lesions associated with decreases in performance in both types of fluency also manifested at the level of left hemispheric white matter tracts: the external capsule, anterior and posterior limbs of the internal capsule, superior and anterior corona radiata and the superior longitudinal fasciculus (Fig. 2B, C, D). No association was found with right hemispheric lesions.

Phonologic fluency scores were specifically associated with lesions of the anterior middle temporal and superior temporal areas, largely the rolandic operculum and the supra-marginal gyrus (fig. 2B, D).

In contrast, semantic fluency scores were specifically associated with lesions of the posterior middle temporal gyrus and largely the pallidum. In addition, lesion to larger portions of the internal capsule and superior longitudinal fasciculus were associated with decreases of semantic than phonologic fluency (fig. 2C, D).

Fig. $1 \& 2$ near here

\section{DISCUSSION}

We localized the brain lesions statistically associated with a decrease in phonologic and semantic verbal fluency performance in a group of 191 unselected patients with a first left or right hemispheric brain damage. The effects of age and education level in each type of fluency and across patients were controlled by using corrected scores for the anatomo-clinical correlations. Our results revealed that the scores in the two types of verbal fluency tasks were positively correlated and that both tasks depended on the integrity of common subcortical areas centered on the left basal ganglia. However, we also found cortical areas preferentially involved in each type of fluency: superior temporal lobe regions for phonologic fluency and middle temporal regions for semantic fluency.

The positive correlation between the scores at the semantic and phonologic verbal fluency tasks suggests that the two tasks involve at least partially shared brain areas. To our knowledge, this is the first 
direct behavioral evidence for a relationship between the scores at the two types of fluency in braindamaged patients. Only few studies measured performance in the two types of fluency in the same neuropsychological population and none of these studies examined whether the performance in the two tasks correlated (Owen et al., 1990, Vilkki and Holst, 1994, Elliott et al., 1996, Baldo and Shimamura, 1998, Stuss et al., 1998, Thomas-Antérion, 1998, Baldo et al., 2001, Baldo et al., 2006, Robinson et al., 2012).

Our finding for more words generated in the semantic than phonologic condition replicates previous evidence for a higher difficulty of phonologic fluency (Ahola et al., 1996, Baldo et al., 2001, Robinson et al., 2012). In our data, we found this pattern in both left and right brain lesion patients, confirming that the lower performance in phonologic fluency was independent on lesion site. Differences in difficulty levels between the two types of fluency have been explained by the fact that retrieving words according to a semantic rule relies on the natural semantic organization of conceptual knowledge; this strategy is thus easier to apply and takes place automatically. By contrast, the search strategy in phonologic fluency requires utilizing unusual and thus slower, more controlled associations based on the first letter of the words (Mummery et al., 1996). Variations in task demands between the two types of fluency have also been demonstrated to account for differences in the brain areas involved in the two tasks, most notably at the level of inferior frontal gyri (Katzef et al., 2013).

Lesions to subcortical brain areas centered on the left basal ganglia decreased performance in both semantic and phonologic fluency. This result for a critical involvement of subcortical structures in verbal fluency contrasts with previous lesion data suggesting that fluency primarily depends on the integrity of cortical structures (Baldo and Shimamura, 1998, Troyer et al., 1998, Schwartz and Baldo, 2001). This discrepancy could be accounted for by the fact that previous studies either focused only on a priori selected cortical regions of interest (Chapados and Petrides, 2013) or did not include patients with subcortical lesion in their analyses (Ahola et al., 1996).

The basal ganglia have been repeatedly involved in executive functions, notably via its interactions with dorsolateral prefrontal regions (DLPFC; Alexander et al., 1986, Mega and Cummings, 1994, Middleton and Strick, 2000). In the fronto-subcortical loop, the DLPFC is connected to the dorsolateral caudate nucleus and the internal globus pallidus via a pathway eventually projecting to the thalamus 
(Alexander et al., 1986). Lesions to the basal ganglia and the thalamus may thus have led to a disruption of the DLPFC activity and in turn impaired verbal fluency (Cox and Heilman, 2011).

This hypothesis is consistent with the finding by Copland and colleagues (2000) that lesions to the basal ganglia impair executive tasks that -as verbal fluency- involve lexical-semantic manipulations, the use of language strategies and the selection between alternative responses to a given linguistic cue (Copland et al., 2000, Crosson et al., 2003). In the same vein, since both types of verbal fluency involve cluster-switching behaviors (i.e. searching within a given field like house pets, and then switch to a new field like aquatic animals (Troyer et al., 1998)), a deficit at this level induced by lesions to the basal ganglia could also account for our VLSM results (e.g Abutalebi et al. 2009; Thames et al., 2012; Troyer et al., 2004).

Finally, lesions to the basal ganglia may have decreased verbal fluency performance by impairing initiation capacities (Pagonabarraga and Kulisevsky, 2012, Del-Monte et al., 2013, Chen et al., 2014). Fabbro et al. (1996) suggested that the left basal ganglia and the thalamus play an important role in regulating arousal and speech initiation, as well as in preverbal semantic monitoring (Crosson, 1985). A disruption of the left DLPFC activity has been shown to result in decreases in switching capacity and in turn in a lack of initiation and perseverations (Troyer et al., 1998). Specifically designed switching task would be helpful to refine our conclusions on this issue.

Shared areas between phonologic and semantic fluency were also evident within ventro-temporal cortical areas. These regions have been previously associated with verbal fluency and are thought to support the storage of semantic knowledge and its role in the associative processes engaged in both tasks (Troster et al., 1998, Troyer et al., 1998).

Together with the high positive correlation between the two tasks in our group of brain-damaged patients, this result for shared subcortical structures suggest that key executive components are similarly involved in fluency tasks, independently on the specific production rules.

In addition to common areas, we found brain regions whose lesions specifically impaired one type of fluency. The integrity of left dorso-lateral temporal areas was necessary for phonologic but not semantic fluency. This dissociation putatively follows from the fact that the word retrieval strategy in phonologic fluency relies more on auditory representations than semantic fluency. Lesions to the Wernicke's area may have impaired preferentially phonologic fluency because it disrupted the phonologic processing loop (e.g Poeppel et al., 2008). These posterior temporal regions belong to the 
associative auditory cortex and mediate the storage of the auditory representation of words (DeWitt and Rauschecker, 2012, Schwartz et al., 2012).

Reversely, lesions to more inferior temporal regions impaired semantic but not phonologic fluency. Inferior temporal regions have been involved in semantic memory and in the "clustering" process likely engaged to find words belonging to the animal semantic category (Troyer et al., 1998). As for the role of occipital regions, it may reflect the fact that the patients relied on a visual mental imagery strategy to retrieve names of animals (D'Esposito et al., 1997). Such dorsal/ventral dissociation between phonological and semantic impairment after brain lesion has been reported in other modalities of language and fits with current general models of language organization (Friederici, 2009, Kummerer et al., 2013).

Dorsal/ventral dissociations have also been found in aphasic patients, where expressive impairments can occur at the lexical-semantic or lexical-phonological levels. Semantic impairments in aphasia can occur at different levels of language processing but have been associated with left temporal, and inferior frontal areas, while lexical-phonological difficulties occur after supramarginal and arcuate lesions (Henseler et al., 2014, Parker Jones et al., 2014). Our results suggest that dorsal/ventral dissociations found in aphasic patients can also be found in an unselected brain-damaged patients population and confirm the role of temporal structure in accessing the meaning of words. While semantic fluency seemed to be more sensitive to lesions of the internal capsule and superior longitudinal fasciculus than phonologic fluency, our results revealed no clear dissociations between the role of ventral and dorsal streams when considering how lesions affected white matter tracts (Saur et al., 2008).

Of note, while the prominent role of left-hemispheric structure in verbal fluency is well known by clinicians for years, by including both left and right hemispheric patients our study provides direct evidence for this assumption (Benton, 1968, Pendleton et al., 1982, Martin et al., 1990, Ahola et al., 1996, Baldo and Shimamura, 1998, Stuss et al., 1998, Troyer et al., 1998, Szatkowska et al., 2000, Baldo et al., 2011, Robinson et al., 2012).

The present study suffers several limitations. First, there were biases in the distribution of strokes due to the differential susceptibility of the cerebral arteries to stroke (Kobayashi et al., 2009) and the fact that some types of strokes increase the probability of meeting an exclusion criterion. For example, left middle and superior frontal gyri lesions were underrepresented in our population because such lesions are often part of major dominant hemispheric syndromes, associated with dementia and severe aphasia (Tatemichi et al., 1993) and with early post stroke depression. These syndromes typically prevent 
neuropsychological testing and likely explain the limited number of such lesions in our population. However, as compared with previous lesion literature on the topic, our study is strongly powered and the lesion distribution still cover left frontal areas. Hence, we feel that this negative result could still be interpreted and suggests that age and education level may account for a part of the involvement of these regions in fluency since the main difference between our and previous lesion studies on fluency is that we used corrected score.

Second, different lesion etiologies were mixed in our VLSM analyses and it cannot be rules out they affected differentially the fluency performance. For example, while Troyer et al. (1998) found the same overall fluency scores in patients with dementia of the Alzheimer type as in those with Parkinson's disease, the two groups differed at the level of the clustering and switching patterns of patients' performance. Recent evidence however indicates that the functional impact of similar lesions but with different etiologies is comparable, which suggests that lesions with different etiologies can be mixed in VLSM analyses (Cipolotti et al., 2015). It is not clear, however, whether the same post-lesional neuroplastic recovery mechanisms take place in each etiology. Tumor being progressive processes and possibly inducing compensatory mechanisms (Yu et al., 2016), and stroke damaging the tissues after very short post-stroke delays, for a given delay after the diagnostic functional reorganization might take place earlier in tumor than stroke patients. Related to this issue, since we focused on data collected during routine neuropsychological assessment, we don't have precise information on which words were produced, which preclude qualitative analyses of e.g. the size and number of cluster across etiologies and lesion sites (Reverberi et al., 2006).

In spite of these limitations, our collective results extend previous literature on verbal fluency by pointing out the critical role of (left) subcortical structures in both phonologic and semantic verbal fluency and a preferential role of dorso-lateral temporal areas in phonologic but not semantic fluency. At the clinical level, our study suggests that fluency tasks should be part of the neurobehavioral evaluation tools to exclude acute brain lesions - particularly to assess the integrity of subcortical nuclei; and that assessing the two types of fluency after subcortical lesions is not necessary since they depend on largely overlapping networks and their scores strongly correlate. However, our study cannot disentangle whether each type of etiology (stroke or tumor) lead to the same impairments and thus the clinical implications mentioned above should be applied with caution. Further studies comparing the fluency 
scores between stroke and tumor patients matched at the level of lesion site are necessary to address this question. 


\section{Figure legends}

Figure 1. Correlation plot of the corrected scores for the phonologic and the semantic verbal fluency task. RHD: Right hemispheric damage; LHD: Left hemispheric damage. The lines represent the linear regressions for the right hemispheric (RHD, red) and the left hemispheric (LHD, green) group of patients.

Figure 2. Voxel-based lesion-symptom mapping results. A. Overlap plot of the lesion of the 191 patients. The number of overlapping lesions is coded with colors ranging from dark red $(n=1)$ to light yellow $(n=31$ patients). B. Voxel-based lesion-symptom mapping (VLSM) on the 191 patients shows that Phonologic fluency impairments were associated with lesions to brain areas centered on left putamen, caudate nucleus and pallidum and left dorsal temporal regions. C. Voxel-based lesion-symptom mapping shows that Semantic fluency impairments were associated with lesions of the left putamen, caudate nucleus and pallidum and left ventral temporal regions. For fig $2 B \&$, only voxels significant at $p<.05$ (FDRcorrected) are color-coded from red $\left(\mathrm{p}_{\mathrm{FDR}}<0.05\right)$ to white $\left(\mathrm{p}_{\mathrm{FDR}}<0.001\right)$. D. Summary of the Voxel-based lesion-symptom mapping results. In red, regions associated with phonologic fluency; in green, with Semantic fluency; in yellow, with both types of fluency. For the figures $2 A-D$, brain slices are displayed from z-coordinates -4 to 32 of the MNI space, with the left hemisphere on the right side. 


\section{References}

Abutalebi J, Rosa PA, Tettamanti M, Green DW, Cappa SF (2009) Bilingual aphasia and language control: a follow-up fMRI and intrinsic connectivity study. Brain and language 109:141-156.

Ahola K, Vilkki J, Servo A (1996) Frontal tests do not detect frontal infarctions after ruptured intracranial aneurysm. Brain and cognition 31:1-16.

Alexander GE, DeLong MR, Strick PL (1986) Parallel organization of functionally segregated circuits linking basal ganglia and cortex. Annual review of neuroscience 9:357-381.

Baldo JV, Schwartz S, Wilkins D, Dronkers NF (2006) Role of frontal versus temporal cortex in verbal fluency as revealed by voxel-based lesion symptom mapping. Journal of the International Neuropsychological Society : JINS 12:896-900.

Baldo JV, Shimamura AP (1998) Letter and category fluency in patients with frontal lobe lesions. Neuropsychology 12:259-267.

Baldo JV, Shimamura AP, Delis DC, Kramer J, Kaplan E (2001) Verbal and design fluency in patients with frontal lobe lesions. Journal of the International Neuropsychological Society : JINS 7:586-596.

Baldo JV, Wilkins DP, Ogar J, Willock S, Dronkers NF (2011) Role of the precentral gyrus of the insula in complex articulation. Cortex; a journal devoted to the study of the nervous system and behavior 47:800-807.

Bates E, Wilson SM, Saygin AP, Dick F, Sereno MI, Knight RT, Dronkers NF (2003) Voxel-based lesionsymptom mapping. Nature neuroscience 6:448-450.

Bechtoldt HPB, A.L; Fogel, M.L (1962) An application of factor analysis in neuropsychology. Psychological Record 12:147-156.

Benke T, Delazer M, Bartha L, Auer A (2003) Basal ganglia lesions and the theory of fronto-subcortical loops: neuropsychological findings in two patients with left caudate lesions. Neurocase 9:70-85.

Benton AL (1968) Differential behavioral effects in frontal lobe disease. Neuropsychologia 53-60

Cardebat D, Doyon B, Puel M, Goulet P, Joanette $Y$ (1990) [Formal and semantic lexical evocation in normal subjects. Performance and dynamics of production as a function of sex, age and educational level]. Acta neurologica Belgica 90:207-217.

Chapados C, Petrides M (2013) Impairment only on the fluency subtest of the Frontal Assessment Battery after prefrontal lesions. Brain : a journal of neurology 136:2966-2978.

Chen JR, Stepanek L, Doupe AJ (2014) Differential contributions of basal ganglia and thalamus to song initiation, tempo, and structure. Journal of neurophysiology 111:248-257.

Cipolotti L, Healy C, Chan E, Bolsover F, Lecce F, White M, Spano B, Shallice T, Bozzali M (2015) The impact of different aetiologies on the cognitive performance of frontal patients. Neuropsychologia 68:21-30.

Copland DA, Chenery HJ, Murdoch BE (2000) Processing lexical ambiguities in word triplets: evidence of lexical-semantic deficits following dominant nonthalamic subcortical lesions. Neuropsychology 14:379-390.

Cox DE, Heilman KM (2011) Dynamic-intentional thalamic aphasia: a failure of lexical-semantic selfactivation. Neurocase 17:313-317.

Crosson $B(1985)$ Subcortical functions in language: a working model. Brain and language 25:257-292.

Crosson B, Benefield H, Cato MA, Sadek JR, Moore AB, Wierenga CE, Gopinath K, Soltysik D, Bauer RM, Auerbach EJ, Gokcay D, Leonard CM, Briggs RW (2003) Left and right basal ganglia and frontal activity during language generation: contributions to lexical, semantic, and phonological processes. Journal of the International Neuropsychological Society : JINS 9:1061-1077. 
D'Esposito M, Detre JA, Aguirre GK, Stallcup M, Alsop DC, Tippet LJ, Farah MJ (1997) A functional MRI study of mental image generation. Neuropsychologia 35:725-730.

Del-Monte J, Capdevielle D, Gely-Nargeot MC, Yazbek H, Pupier F, Boulenger JP, Raffard S (2013) [Evolution of the concept of apathy: the need for a multifactorial approach in schizophrenia]. L'Encephale 39 Suppl 1:S57-63.

DeWitt I, Rauschecker JP (2012) Phoneme and word recognition in the auditory ventral stream. Proceedings of the National Academy of Sciences of the United States of America 109:E505-514.

Elliott R, Sahakian BJ, McKay AP, Herrod JJ, Robbins TW, Paykel ES (1996) Neuropsychological impairments in unipolar depression: the influence of perceived failure on subsequent performance. Psychological medicine 26:975-989.

Fabbro F, Clarici A, Bava A (1996) Effects of left basal ganglia lesions on language production. Perceptual and motor skills 82:1291-1298.

Fiez JA, Damasio H, Grabowski TJ (2000) Lesion segmentation and manual warping to a reference brain: intra- and interobserver reliability. Human brain mapping 9:192-211.

Friederici AD (2009) Pathways to language: fiber tracts in the human brain. Trends in cognitive sciences 13:175-181.

Fu CH, Morgan K, Suckling J, Williams SC, Andrew C, Vythelingum GN, McGuire PK (2002) A functional magnetic resonance imaging study of overt letter verbal fluency using a clustered acquisition sequence: greater anterior cingulate activation with increased task demand. Neurolmage 17:871-879.

Gourovitch ML, Kirkby BS, Goldberg TE, Weinberger DR, Gold JM, Esposito G, Van Horn JD, Berman KF (2000) A comparison of rCBF patterns during letter and semantic fluency. Neuropsychology 14:353-360.

Grossman M (1981) A bird is a bird is a bird: making reference within and without superordinate categories. Brain and language 12:313-331.

Henry JD, Crawford JR (2004) A meta-analytic review of verbal fluency performance following focal cortical lesions. Neuropsychology 18:284-295.

Henseler I, Regenbrecht F, Obrig H (2014) Lesion correlates of patholinguistic profiles in chronic aphasia: comparisons of syndrome-, modality- and symptom-level assessment. Brain : a journal of neurology 137:918-930.

Hodges JR, Salmon DP, Butters N (1992) Semantic memory impairment in Alzheimer's disease: failure of access or degraded knowledge? Neuropsychologia 30:301-314.

Katzev M, Tuscher O, Hennig J, Weiller C, Kaller CP (2013) Revisiting the functional specialization of left inferior frontal gyrus in phonological and semantic fluency: the crucial role of task demands and individual ability. The Journal of neuroscience : the official journal of the Society for Neuroscience 33:7837-7845.

Kobayashi A, Wardlaw JM, Lindley RI, Lewis SC, Sandercock PA, Czlonkowska A, Group ISTC (2009) Oxfordshire community stroke project clinical stroke syndrome and appearances of tissue and vascular lesions on pretreatment ct in hyperacute ischemic stroke among the first 510 patients in the Third International Stroke Trial (IST-3). Stroke; a journal of cerebral circulation 40:743-748.

Kummerer D, Hartwigsen G, Kellmeyer P, Glauche V, Mader I, Kloppel S, Suchan J, Karnath HO, Weiller C, Saur D (2013) Damage to ventral and dorsal language pathways in acute aphasia. Brain : a journal of neurology 136:619-629.

Lawrence AD, Sahakian BJ, Robbins TW (1998) Cognitive functions and corticostriatal circuits: insights from Huntington's disease. Trends in cognitive sciences 2:379-388.

Manuel AL, Radman N, Mesot D, Chouiter L, Clarke S, Annoni JM, Spierer L (2013) Inter- and intrahemispheric dissociations in ideomotor apraxia: a large-scale lesion-symptom mapping study in subacute brain-damaged patients. Cereb Cortex 23:2781-2789. 
Marsolais Y, Methqal I, Joanette Y (2015) Marginal neurofunctional changes in high-performing older adults in a verbal fluency task. Brain and language 140:13-23.

Martin A, Wiggs CL, Ungerleider LG, Haxby JV (1996) Neural correlates of category-specific knowledge. Nature 379:649-652.

Martin R, Loring DW, Meador KJ, Lee GP (1990) The effects of lateralized temporal lobe dysfunction on formal and semantic word fluency. Neuropsychologia 28:823-829.

Mega MS, Cummings JL (1994) Frontal-subcortical circuits and neuropsychiatric disorders. The Journal of neuropsychiatry and clinical neurosciences 6:358-370.

Middleton FA, Strick PL (2000) Basal ganglia and cerebellar loops: motor and cognitive circuits. Brain research Brain research reviews 31:236-250.

Miyake A, Friedman NP, Emerson MJ, Witzki AH, Howerter A, Wager TD (2000) The unity and diversity of executive functions and their contributions to complex "Frontal Lobe" tasks: a latent variable analysis. Cognitive psychology 41:49-100.

Moscovitch M (1994) Cognitive resources and dual-task interference effects at retrieval in normal people: The role of the frontal lobes and medial temporal cortex. Neuropsychology 8:524-534.

Mummery CJ, Patterson K, Hodges JR, Wise RJ (1996) Generating 'tiger' as an animal name or a word beginning with $\mathrm{T}$ : differences in brain activation. Proceedings Biological sciences / The Royal Society 263:989-995.

Owen AM, Downes JJ, Sahakian BJ, Polkey CE, Robbins TW (1990) Planning and spatial working memory following frontal lobe lesions in man. Neuropsychologia 28:1021-1034.

Pagonabarraga J, Kulisevsky J (2012) Cognitive impairment and dementia in Parkinson's disease. Neurobiology of disease 46:590-596.

Parker Jones O, Prejawa S, Hope TM, Oberhuber M, Seghier ML, Leff AP, Green DW, Price CJ (2014) Sensory-to-motor integration during auditory repetition: a combined fMRI and lesion study. Frontiers in human neuroscience 8:24.

Pendleton MG, Heaton RK, Lehman RA, Hulihan D (1982) Diagnostic utility of the Thurstone Word Fluency Test in neuropsychological evaluations. Journal of clinical neuropsychology 4:307-317.

Phelps EA, Hyder F, Blamire AM, Shulman RG (1997) FMRI of the prefrontal cortex during overt verbal fluency. Neuroreport 8:561-565.

Poeppel D, Idsardi WJ, van Wassenhove V (2008) Speech perception at the interface of neurobiology and linguistics. Philosophical transactions of the Royal Society of London Series B, Biological sciences 363:1071-1086.

Pujol J, Vendrell P, Deus J, Kulisevsky J, Marti-Vilalta JL, Garcia C, Junque C, Capdevila A (1996) Frontal lobe activation during word generation studied by functional MRI. Acta neurologica Scandinavica 93:403-410.

Reverberi C, Laiacona M, Capitani E (2006) Qualitative features of semantic fluency performance in mesial and lateral frontal patients. Neuropsychologia 44:469-478.

Robinson G, Shallice T, Bozzali M, Cipolotti L (2012) The differing roles of the frontal cortex in fluency tests. Brain : a journal of neurology 135:2202-2214.

Rorden C, Brett M (2000) Stereotaxic display of brain lesions. Behavioural neurology 12:191-200.

Rorden C, Karnath HO, Bonilha L (2007) Improving lesion-symptom mapping. Journal of cognitive neuroscience 19:1081-1088.

Ruff RM, Light RH, Parker SB, Levin HS (1997) The psychological construct of word fluency. Brain and language 57:394-405.

Saur D, Kreher BW, Schnell S, Kummerer D, Kellmeyer P, Vry MS, Umarova R, Musso M, Glauche V, Abel S, Huber W, Rijntjes M, Hennig J, Weiller C (2008) Ventral and dorsal pathways for language. Proceedings of the National Academy of Sciences of the United States of America 105:1803518040. 
Schwartz MF, Faseyitan O, Kim J, Coslett HB (2012) The dorsal stream contribution to phonological retrieval in object naming. Brain : a journal of neurology 135:3799-3814.

Schwartz S, Baldo J (2001) Distinct patterns of word retrieval in right and left frontal lobe patients: a multidimensional perspective. Neuropsychologia 39:1209-1217.

Shapira-Lichter I, Oren N, Jacob Y, Gruberger M, Hendler T (2013) Portraying the unique contribution of the default mode network to internally driven mnemonic processes. Proceedings of the National Academy of Sciences of the United States of America 110:4950-4955.

Spierer L, Bellmann-Thiran A, Maeder P, Murray MM, Clarke S (2009) Hemispheric competence for auditory spatial representation. Brain : a journal of neurology 132:1953-1966.

Stuss DT, Alexander MP, Hamer L, Palumbo C, Dempster R, Binns M, Levine B, Izukawa D (1998) The effects of focal anterior and posterior brain lesions on verbal fluency. Journal of the International Neuropsychological Society : JINS 4:265-278.

Szatkowska I, Grabowska A, Szymanska O (2000) Phonological and semantic fluencies are mediated by different regions of the prefrontal cortex. Acta neurobiologiae experimentalis 60:503-508.

Tatemichi TK, Desmond DW, Paik M, Figueroa M, Gropen TI, Stern Y, Sano M, Remien R, Williams JB, Mohr JP, et al. (1993) Clinical determinants of dementia related to stroke. Annals of neurology 33:568-575.

Thames AD, Foley JM, Wright MJ, Panos SE, Ettenhofer M, Ramezani A, Streiff V, El-Saden S, Goodwin S, Bookheimer SY, Hinkin CH (2012) Basal ganglia structures differentially contribute to verbal fluency: evidence from Human Immunodeficiency Virus (HIV)-infected adults. Neuropsychologia 50:390-395.

Thomas-Antérion CG, F.; Ozanne, B.; Laurent, B. (1998) Etude quantitative et qualitative de l'évocation lexicale dans les démences frontotemporale et d'Alzheimer. Revue de neuropsychologie 8:415430.

Thompson-Schill SL, D'Esposito M, Aguirre GK, Farah MJ (1997) Role of left inferior prefrontal cortex in retrieval of semantic knowledge: a reevaluation. Proceedings of the National Academy of Sciences of the United States of America 94:14792-14797.

Thompson-Schill SL, Swick D, Farah MJ, D'Esposito M, Kan IP, Knight RT (1998) Verb generation in patients with focal frontal lesions: a neuropsychological test of neuroimaging findings. Proceedings of the National Academy of Sciences of the United States of America 95:1585515860.

Thuillard F, Assal G (1991) Données neuropsychologiques chez le sujet âgé normal. In: Démences et syndromes démentiels : approche neuropsychologique(Habib, M., Joanette, Y., Pue, I., M., éds., ed), pp 125-133 Paris: Masson.

Tombaugh TN, Kozak J, Rees L (1999) Normative data stratified by age and education for two measures of verbal fluency: FAS and animal naming. Archives of clinical neuropsychology : the official journal of the National Academy of Neuropsychologists 14:167-177.

Troster Al, Fields JA, Testa JA, Paul RH, Blanco CR, Hames KA, Salmon DP, Beatty WW (1998) Cortical and subcortical influences on clustering and switching in the performance of verbal fluency tasks. Neuropsychologia 36:295-304.

Troyer AK, Black SE, Armilio ML, Moscovitch M (2004) Cognitive and motor functioning in a patient with selective infarction of the left basal ganglia: evidence for decreased non-routine response selection and performance. Neuropsychologia 42:902-911.

Troyer AK, Moscovitch M, Winocur G, Alexander MP, Stuss D (1998) Clustering and switching on verbal fluency: the effects of focal frontal- and temporal-lobe lesions. Neuropsychologia 36:499-504.

Vilkki J, Holst P (1994) Speed and flexibility on word fluency tasks after focal brain lesions. Neuropsychologia 32:1257-1262. 
Wagner AD, Pare-Blagoev EJ, Clark J, Poldrack RA (2001) Recovering meaning: left prefrontal cortex guides controlled semantic retrieval. Neuron 31:329-338.

Wilke M, de Haan B, Juenger H, Karnath HO (2011) Manual, semi-automated, and automated delineation of chronic brain lesions: a comparison of methods. Neurolmage 56:2038-2046.

Table 1. Detailed demographic information

\begin{tabular}{|c|c|c|c|c|c|c|}
\hline & Group size & \multicolumn{2}{|c|}{ Sexe } & \multicolumn{2}{c|}{ Damaged hemisphere } & \multirow{2}{*}{$\begin{array}{c}\text { Education level } \\
\end{array}$} \\
\hline & male & female & Left & Right & $(/ 3)$ \\
\hline Stroke & 134 & 91 & 43 & 77 & 57 & 1,7 \\
\hline Tumor & 57 & 29 & 28 & 31 & 26 & 1,9 \\
\hline
\end{tabular}


Figure 1.

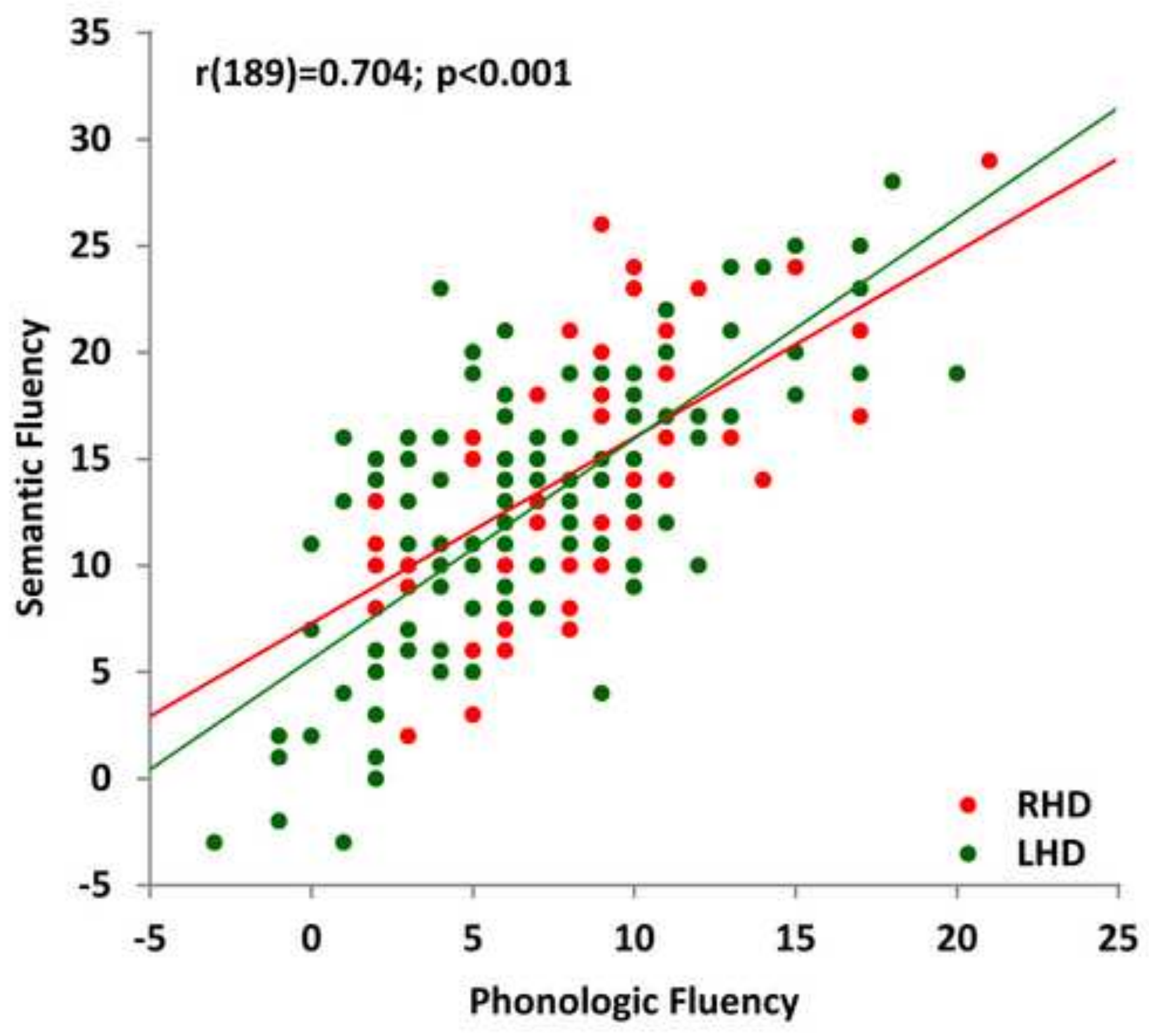


Figure 2

A Lesions Overlay Of The 191 Patients

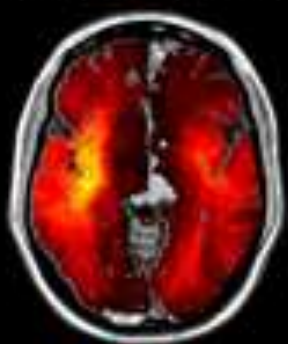

$z=-4$

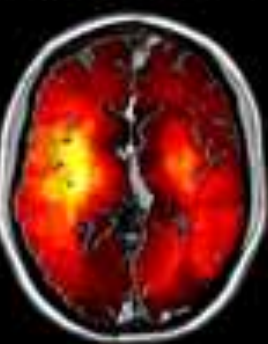

4

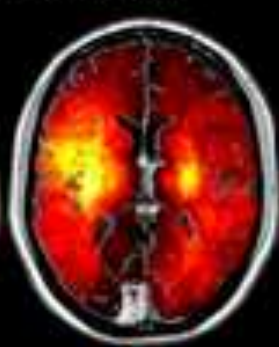

12

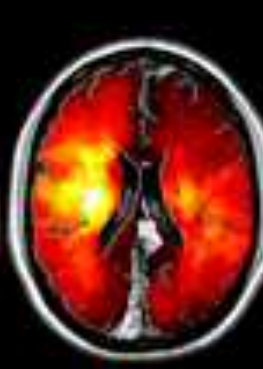

20
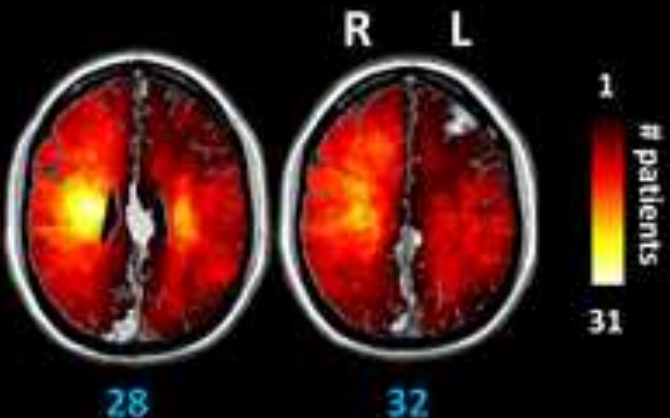

B Phonologic Verbal Fluency Task VLSM

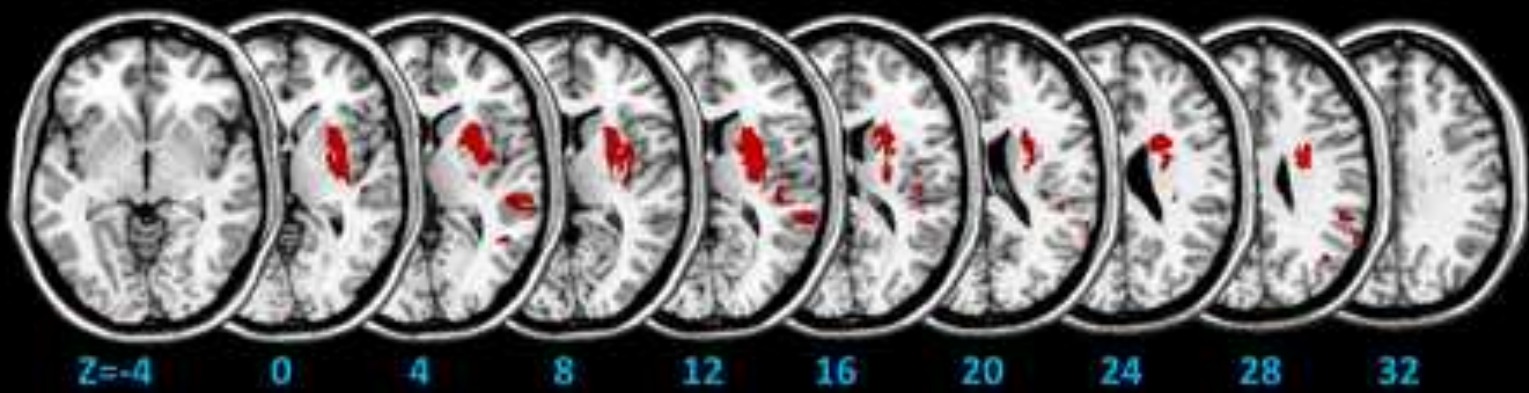

C Semantic Verbal Fluency Task VLSM

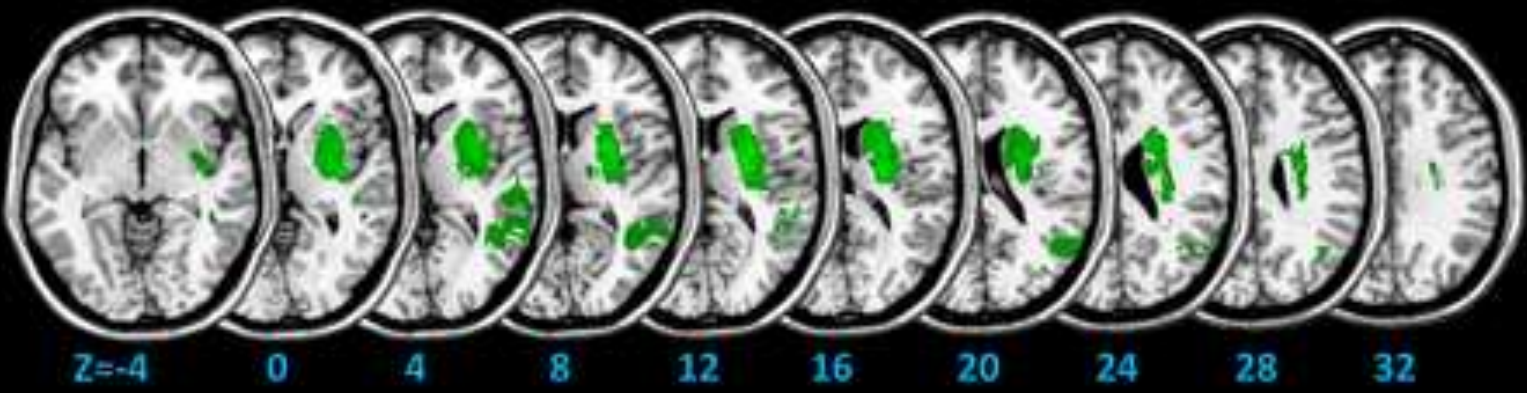

D Overlap Between The Phonologic And Semantic Tasks
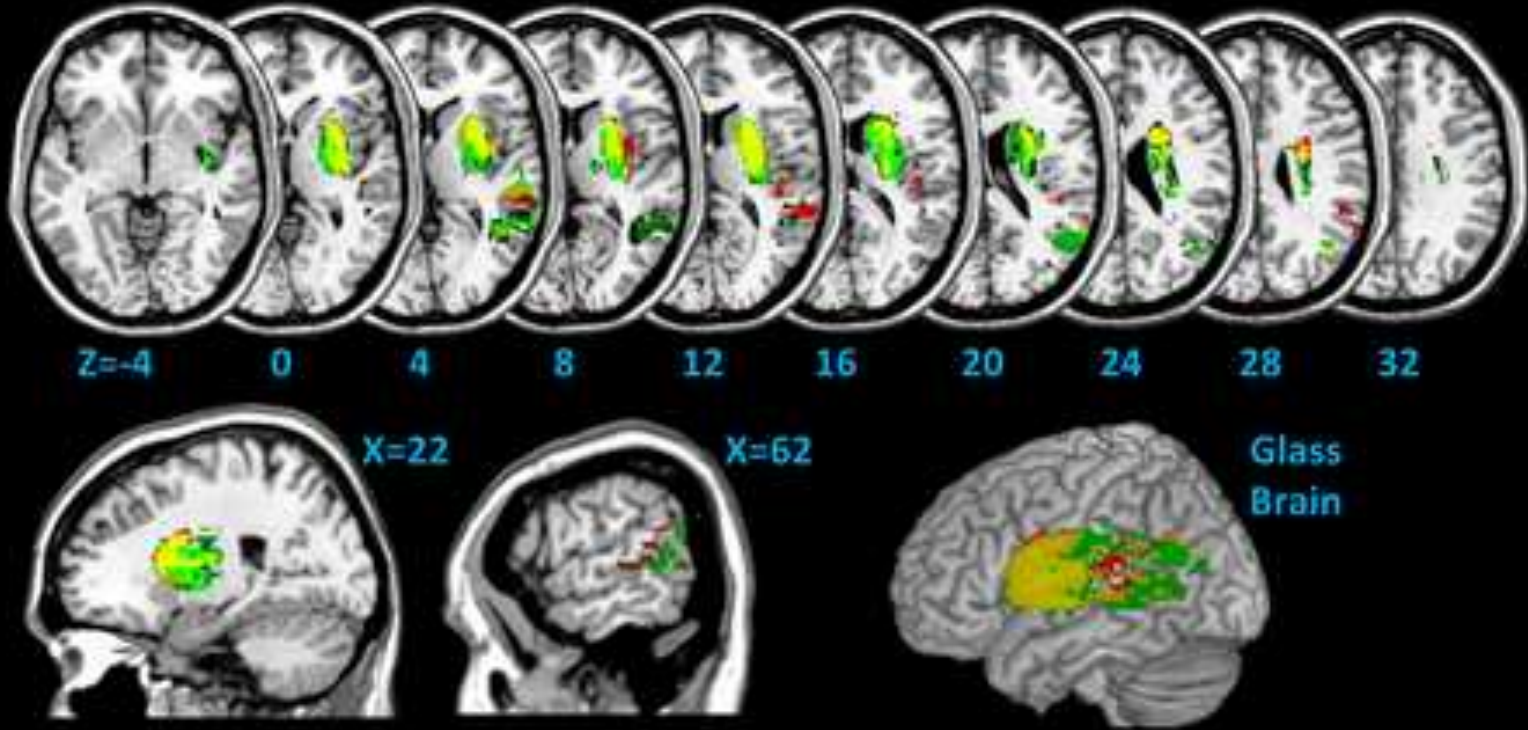

$P_{F D R}<0.05$

$P_{F D R}<0.05$

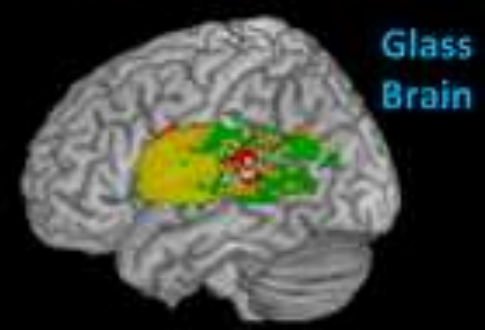

Phonologic

Fluency

Semantic

Fluency

Both 